\title{
Real-time Feedback for Improving Medication Taking
}

\author{
Matthew L. Lee \\ Philips Research North America \\ Briarcliff Manor, NY, USA \\ matthew.lee@philips.com
}

\author{
Anind K. Dey \\ HCI Institute, Carnegie Mellon University \\ Pittsburgh, PA, USA \\ anind@cs.cmu.edu
}

\begin{abstract}
Medication taking is a self-regulatory process that requires individuals to self-monitor their medication taking behaviors, but this can be difficult because medication taking is such a mundane, unremarkable behavior. Ubiquitous sensing systems have the potential to sense everyday behaviors and provide the objective feedback necessary for self-regulation of medication taking. We describe an unobtrusive sensing system consisting of a sensor-augmented pillbox and an ambient display that provides near real-time visual feedback about how well medications are being taken. In contrast to other systems that focus on reminding before medication taking, our approach uses feedback after medication taking to allow the individual to develop their own routines through selfregulation. We evaluated this system in the homes of older adults in a 10-month deployment. Feedback helped improve the consistency of medication-taking behaviors as well as increased ratings of self-efficacy. However, the improved performance did not persist after the feedback display was removed, because individuals had integrated the feedback display into their routines to support their self-awareness, identify mistakes, guide the timing of medication taking, and provide a sense of security that they are taking their medications well. Finally, we reflect on design considerations for feedback systems to support the process of self-regulation of everyday behaviors.
\end{abstract}

\section{Author Keywords}

Medication adherence, sensors, feedback, behavior change, self-efficacy, ambient display, self-regulation

\section{ACM Classification Keywords}

H.5.m. Information interfaces and presentation (e.g., HCI): Miscellaneous.

\section{INTRODUCTION}

Over two-thirds of people over the age of 60 take four or more medications on a daily basis, and those with multiple chronic conditions take nine or more daily medications [6]. However, former U.S. surgeon general C. Everett Koop famously quipped, "Drugs don't work in patients who don't take them." Approximately $20-50 \%$ of patients do not take

Permission to make digital or hard copies of all or part of this work for personal or classroom use is granted without fee provided that copies are not made or distributed for profit or commercial advantage and that copies bear this notice and the full citation on the first page. Copyrights for components of this work owned by others than the author(s) must be honored. Abstracting with credit is permitted. To copy otherwise, or republish, to post on servers or to redistribute to lists, requires prior specific permission and/or a fee. Request permissions from Permissions@acm.org. CHI 2014, April 26 - May 01 2014, Toronto, ON, Canada

Copyright is held by the owner/author(s). Publication rights licensed to ACM.

ACM 978-1-4503-2473-1/14/04 ..\$15.00.

http://dx.doi.org/10.1145/2556288.2557210 at least $80 \%$ of their prescribed medications [13]. Improving medication adherence can lead to improved health outcomes and decreased health care costs [19].

Medication non-adherence comes in two forms: intentional (e.g., due to inconvenience or fear of side effects) and unintentional (e.g., due to being forgetful or careless). Much attention has been devoted to interventions to reduce intentional non-adherence by motivating individuals using complex programs involving education, individual attention from a clinician, counseling, and rewards [13]. However, unintentional non-adherence due to forgetfulness or carelessness is still pervasive, with $70 \%$ of a large sample of people with chronic diseases reporting instances of unintentional non-adherence [10].

Reminder systems such as MEMS [28] and CAMP [17] can provide alerts for medication taking. However, they can interfere with the individual's routines, do not reinforce the individual to self-initiate, and may undermine the individual's autonomy, an important mediator for medication taking [26]. Thus, there is a need for interventions for individuals already motivated to take their medications that do not adversely affect their autonomy.

Motivated individuals engage in self-regulation to adhere to their medication regimen by setting goals, actively pursuing these goals, and checking their progress or attainment of their goals [18]. Individuals must have feedback about their behaviors to compare their current behavior and their goal behavior and make adjustments to be more consistent. However, reflecting on medication taking is difficult because it is such an uninteresting, routine task performed every day. As a result, mentally tracking and self-reporting of medication adherence tends to be inaccurate [11].

Technologies such as sensors and ambient displays embedded in the home can provide the objective feedback necessary for engaging in self-regulation. These solutions can sense how the individual performs their medication taking and provide information about missed, late, or incorrectly taken medications. The information can also be delivered in a timely and locationally appropriate context that actually allows the individual to compare their current performance with their desired performance and adjust to perform better.

In this paper, we investigate how providing real-time sensor-based feedback about medication taking affects how consistently individuals take their medications. We describe 
a sensing and feedback system consisting of 1) a sensoraugmented pillbox that objectively monitors the individual's medication taking behavior and 2) an in-home ambient display that provides visual feedback about how well medications were taken for the current day. We deployed this system in the homes of older adults who take multiple daily medications in a longitudinal study for 10 months to examine the effect of feedback on medication taking behavior over time.

We demonstrate how the addition of a real-time feedback display helped individuals improve the promptness, correctness, and reduce the variability of their medication taking, when compared to their own baseline without the feedback display, and when compared to a control group who did not receive the real-time feedback display. The sensor-based feedback about successes and failures in medication taking also improved ratings of self-efficacy. In the next section, we describe related work before introducing the system, deployment study, and results.

\section{RELATED WORK}

Medication adherence review studies [12,13] show that clinical interventions with the largest effect sizes involve dosage simplification or combinations of counseling, reminders, follow up, supervised self-monitoring, and feedback. To avoid unintentional non-adherence due to carelessness or forgetfulness (a common issue for both older adults or busy younger adults) [10], motivated individuals need to be equipped with the skills to carry out their intention to take their medications.

One way to provide external assistance for medication taking is to use reminders. For example, a weekly phone call from a nurse can improve adherence rates [9]. Simple reminders like the GlowCap [28] as well as intelligent, context-aware reminders [11] based on multiple sensors around the home can also improve adherence rates. However reminders can be intrusive or heavy-handed, particularly for individuals who believe they take their medications well and do not need a reminder [20]. Reminders also do not fit well into the self-regulation process used to adopt a new behavior because they do not provide feedback for refining the behavior. Another approach for providing assistance for medication taking is to transform it from being a burdensome task to a social or entertaining task using a mobile game [23] with other medication takers. Our approach aims to be more lightweight, supporting the patient's autonomy without imposing a predefined routine for medication taking. We unobtrusively collect and present objective behavioral information to empower individuals to self-initiate their medication taking on their own using their natural process of self-regulation.

Self-Regulation Theory [2] provides a framework to understand the process of how individuals use contextual information to make decisions and follow through on intentions for behavior change. According to Self-
Regulation Theory, human behavior is "regulated by the continual exercise of self-influence" [2]. To engage in selfregulation, individuals must 1) self-monitor their own behaviors and determinants of these behaviors, 2) make judgments about their behaviors with respect to personal standards, and 3) effectively self-reinforce their behaviors. Within this framework, feedback systems are well suited to help individuals self-monitor their behaviors more objectively and more easily than previously possible and to deliver this information in a way that allows them to draw comparisons with their goal behaviors.

Medication adherence is a self-regulatory process, where the individual sets a mental goal to take their medications, attempts to take their medications consistently, selfmonitors how consistent they have been, and self-regulates their attitudes and behaviors to reach their adherence goal. Moreover, goal setting [16], a technique explored in other technologies for behavior change (e.g., [5]), also facilitates the process of behavior change. In particular, frequent feedback about achieving sub-goals can make the sub-goals feel more tangible and also shows progress towards the larger overall goal.

An individual who experiences success in reaching a subgoal such as taking his morning medications correctly today may also increase his self-efficacy [1], the feeling of confidence that he can perform his medication taking well despite obstacles such as being busy or forgetful. High selfefficacy is predictive of better performance, as long as the individual has the feedback to recognize his successes and failures and adjust accordingly to improve his medicationtaking performance.

Feedback to the individual in clinical studies has usually involved manual self-recording of medication taking with diaries and have been combined with other interventions with limited success [13]. In the HCI domain, automated behavioral feedback has been useful in other behaviors that depend on self-regulation, such as performing physical activity [4,15], reducing resource consumption [7] and maintaining self-awareness [14]. In our work, we also use an approach for unobtrusively and objectively sensing medication-taking behavior that does not require the individual to do anything differently. We reflect their behaviors back to the individual with an in-home ambient display to determine whether the feedback helps individuals improve the consistency of their medication taking. This reflection approach follows a tradition of using ambient displays $[4,25]$ that afford informational feedback at a quick glance to facilitate behavior change.

\section{RESEARCH QUESTIONS}

In this paper, we set out to answer the following questions:

1. How does real-time feedback help individuals improve the consistency of their medication-taking behaviors?

2. How does real-time feedback affect self-efficacy for medication taking? 
3. How do medication-taking behaviors change when removing real-time feedback after long-term use?

To answer these questions, we designed, built, and deployed a system for sensing and providing near real-time feedback for medication taking and evaluated this system using a 10-month longitudinal study to identify the effects of feedback on medication-taking behaviors.

\section{SENSING AND FEEDBACK SYSTEM}

To track medication taking unobtrusively, we designed and built a custom pillbox sensor. Commercial MEMS trackers like the Vitality GlowCap [28] were not used in this study because participants used weekly pillboxes (not pill bottles) in their medication taking routines. GlowCaps also uses reminders instead of providing real-time feedback for selfregulation. Instead, we augmented a 7-day pillbox [Figure 2] commonly found in drug stores with 1) a 3-axis accelerometer to track when the pillbox is picked up and 2) spring switches on the doors to track when the doors are opened and 3) custom circuitry with PICAXE microcontrollers and a XBee wireless modem. The design of the pillbox was similar to approaches used by Fishkin [8] and Hayes [11], but was wireless and more easily integrated into the user's routines. The pillbox sensor transmitted its data to a laptop in the participant's home using a ZigBeewireless network. The laptop uploaded data periodically to a remote server where the sensor data was processed to identify and assess episodes of medication taking. A pilltaking episode consists of opening the correct pillbox door and inverting the pillbox to pour out the pills. While it is true that the sensor cannot actually detect whether the individual has swallowed the pills, the sensor can still capture the important step of remembering when and which pills to take to avoid unintentional non-adherence. The pillbox battery lasted between 4 to 6 weeks before needing to be replaced by a researcher.

The sensor-augmented pillbox was one sensor in a suite of sensors in the dwellSense system deployed in participants' homes which comprised other sensors to track Instrumental Activities of Daily Living, including motion/seat sensors, a sensor to track phone misdials, and an augmented coffee maker to track the routine, cognitively-demanding task of making coffee. In this paper, we focus only on medication taking because it is a widely recognized problem.

Near real-time feedback about medication taking was presented on a tablet display placed in the home of the individual. The display used a 10" Samsung Galaxy Tab running a custom application that shows a dashboard view [Figure 1] of how well the individual carried out her medication taking, phone use, and coffee making so far for the current day. The top row shows the status of medication taking for the morning and evening episodes including the time of day, which pillbox doors they opened, and whether they were taken on time, late, or not at all. We asked each individual 1) what time of day they normally take their morning and evening pills and 2) what time of day they

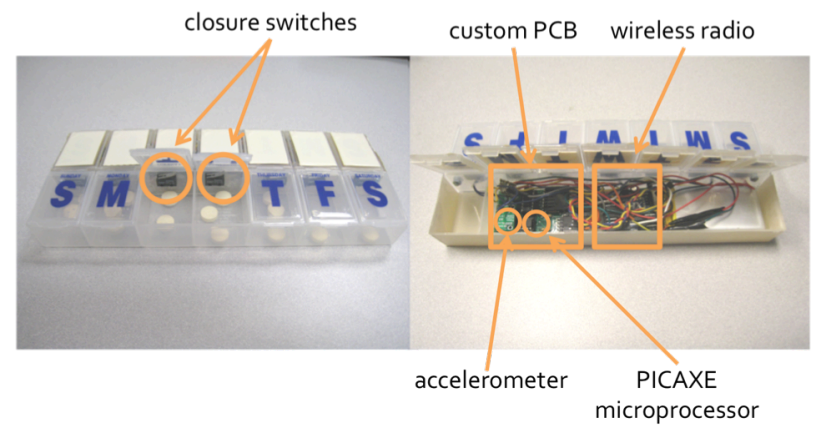

Figure 2. Sensor-augmented pillbox unobtrusively monitored medication taking over 10 months.

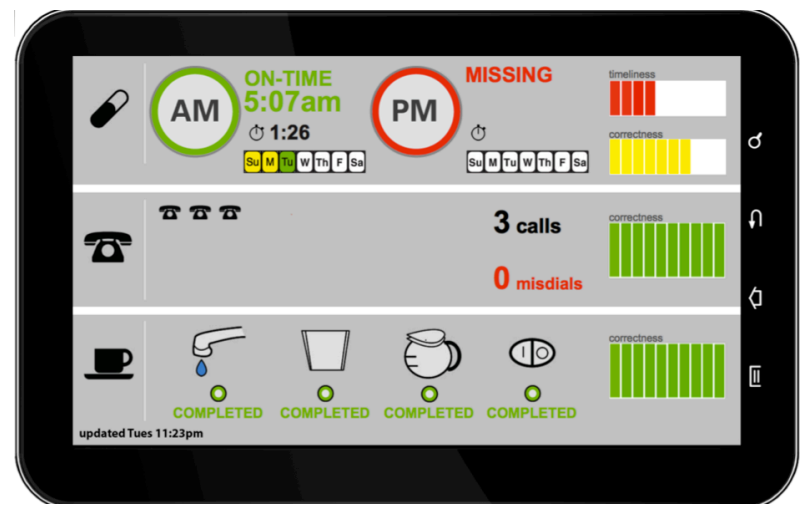

Figure 1. An in-home tablet-based display shows feedback about how well individuals carry out their medicationtaking, phone use, and coffee making. It shows information about what time meds were taken, whether it was on-time, late or missing, what pillbox doors were opened, and a glanceable visual rating (right) for the promptness and correctness of medication taking.

would consider it to be late but still acceptable to take their pills. Users typically set the "late time" to be at least three hours after their normal pill taking time.

The status for the AM and PM medication taking episodes would light up in green with the text "ON-TIME" if pills were taken before the user-defined late time. The status changes to red with the text "MISSING" if the pills were not taken by the user-defined late time. The display changes to yellow with the text "LATE" if they took their pills after the late time. The bars on the right show a glanceable rating for promptness and correctness (opening the correct pillbox door to match the current day). The promptness bar shows all 10 bars if both AM and PM episodes are taken on-time, only 7 bars if either episodes are late, and at most 5 bars if either episodes are missing. The correctness bar shows all 10 bars if only the correct door was opened in both episodes, 7 bars if an extra door was opened, and at most 5 bars if the correct door was not opened. The display updates itself approximately once every 30 minutes to prevent displayed values from oscillating too quickly as tasks were being performed, which could be perceived as distracting or confusing. At the end of the day while the individual was asleep, the values of the display were automatically re- 
initialized, so users would see only the activity reports for the current day. Aside from pressing the "back" button that showed the report from the previous day, the display required no other user input and acted as an always-on ambient display. All other features on the tablet were disabled. The visual design of the display was pilot-tested with three older adults for two weeks and was revised based on user feedback before the subsequent deployment.

\section{DEPLOYMENT STUDY}

Participants were recruited from a local senior apartment building for people over 55 years old. The inclusion criteria included individuals who took multiple medications (pills) daily and were living alone. From 34 who expressed interest in the study, 12 older adults ( 9 female, 3 male) were willing to use the pillbox sensor and were enrolled in the study. The sample's age range was 52 to 83 (mean of 67.5) years. Each had multiple chronic conditions (averaging 2.5 conditions), such as diabetes, arthritis, hypertension, and COPD. All participants initially reported that they were taking their medications consistently every day. Thus, none felt they needed assistance to improve their medication taking but still had the overall goal to take their medications consistently. Participants were given a $\$ 30$ grocery gift card each month for participating in the study. The gift card was not tied to their medication-taking performance.

Each individual was assigned randomly to either the feedback group or control group. Both groups had their medication taking monitored with the sensor-augmented pillbox. The six participants in the feedback group received the tablet display that showed real-time feedback on their task performance. The remaining six participants were assigned to the control group and never received the tablet display. There were no statistically significant differences $(p>0.10)$ in age, number of chronic conditions, and gender between the two groups as measured by Student's t-tests.

\section{Deployment}

To capture the impact of real-time feedback on medicationtaking behaviors, the pillbox sensor was installed in the homes of the participants for 10 months. According to their existing routine, participants kept the pillbox either in the living room, kitchen, bedroom, or bathroom. A researcher visited on average once or twice a month to check on the sensors, replace batteries, and administer questionnaires throughout the study.

Months 1 and 2 of the study formed the baseline period for both the control and feedback groups in which no feedback was presented to participants. The feedback group received the tablet display in the beginning of month 3 , providing them with real-time feedback on their medication taking. Each member of this group chose to keep the display in the living room where they spent most of their time. We provided an instruction guide on how to read the display, walked through the display with the participant, and tested the participant's comprehension of the data values before completing the visit. We did not prescribe exactly how often and when the participant should look at the display, but recommended that the participant look at the display whenever they wanted to, with suggested examples including before or after their medication-taking time. We also emphasized that the point of the study was NOT that they should take their medications any differently unless that was something they wanted to do for themselves. In fact, the researchers directed the participant's attention to reporting any errors they may see in the display, for example when the display did not match their behavior.

After 6 months of deployment (end of month 8), the display was removed from the homes of the feedback group. The pillbox sensors continued to track their medication taking behaviors for 2 months following the removal of the tablet.

The control group never received the tablet display. Instead, at the end of the month 4 , the control group received a paper printout showing when they had taken their medications during the months 3 and 4 of the study. We presented this limited, one-time feedback to ensure that both groups would be familiar with the type of data being collected by the pillbox sensor. Thus, both groups knew exactly how their behaviors were being monitored.

At the end of month 10, all the sensors were removed from the participants' homes. Once a month over the entire study period, researchers used verbal questionnaires to assess each participant's self-efficacy in medication taking.

\section{Measures}

We consider the effect of real-time feedback on the consistency of medication-taking behaviors as captured by the sensor data, and self-efficacy to take medications as captured by verbal questionnaires.

\section{Measures of Medication-taking Consistency}

We consider the following important features of medication taking for our analysis: adherence, correctness, promptness, and the variance in the time of day the pills were taken.

Medication adherence characterizes how often pills are taken and is calculated as a percentage by summing all the pill-taking instances when the individual has taken their pills and dividing the sum by the prescribed number of instances in a given time period.

Correctness characterizes whether the individual took the pills assigned for that particular day. A pill-taking instance was considered correct if the individual opened up the pillbox door that matched the current day of the week. Users could open up any other doors in addition to the door that matched the current day and the instance would still be considered correct because it is likely they took the correct pill after realizing they opened the wrong door(s).

It is recommended to take medications (e.g., for managing blood pressure [27]) at the proper time to ensure a steady level of medication in the body, so we consider two measures of timeliness: promptness and variance. 
Promptness characterizes whether the pills are taken before the user-specified time-of-day threshold for late pills. We asked each participant to specify a time of day that they considered later than they normally would take their morning and evening pills. The rate of promptness was calculated as a percentage by summing the number of pilltaking instances before the late time and dividing it by the total number of instances taken over a given period of time.

Variance in the time of day measures how the time of day that medications were taken varied from one day to another. Lower variance is better because it spreads out the dosages more evenly and ensures that the level of medication in the individual's system remains more consistent from day to day. The variance was calculated by converting the time of day to a decimal number between 0 and 24 and then taking the variance of the numeric times over a given time period.

\section{Measure of Self-Efficacy}

To measure the individual's self-efficacy in taking medications over time, once every month a researcher verbally administered the Self-Efficacy for Appropriate Medication Use Scale (SEAMS), a validated 13-item questionnaire [24]. Each item in the questionnaire presented a potentially challenging situation (such as encountering side effects, having a busy day, or taking pills multiple times a day) and had the respondent rate their confidence as "Not Confident", "Somewhat Confident", and "Very Confident" in these situations.

\section{RESULTS}

Based on 5,785 episodes of medication taking by 12 older adults over 10 months, we investigate how real-time feedback affects medication-taking behaviors and selfefficacy. Participant P04 (control group) withdrew from the study after month 5 because he moved out of his apartment. During the initial 2 months (the baseline period) before any intervention, the groups were equivalent and had no significant differences in measures of medication-taking adherence $(p=0.649)$, promptness $(p=0.610)$, correctness $(p=0.804)$, variance in the time of day taken $(p=0.714)$, or ratings of self-efficacy $(\mathrm{p}=0.614)$ as measured by t-tests.

Participants with the display reported that they looked at the display at least once or twice a day, with most reporting they looked at it periodically throughout the day because "it was there." Participants found the display to be relatively easy to interpret, with the exception of the [Su M T W Th F $\mathrm{Sa}$ ] correctness display for each episode. They initially interpreted it as showing their progress for the current week, when in fact, it shows which doors were opened for today's AM or PM episode. After a researcher clarified it, participants understood the correctness display.

\section{Data Analysis Procedure}

For the analysis of behavior change, we focus on whether the real-time feedback display had an effect, as compared to both the control group's behavior and also the feedback group's own baseline (to account for individual differences). The rate of adherence, rate of promptness, rate of correctness, and variance in the time of day were calculated for each month for a repeated-measures analysis.

The first 2 months represent a Baseline phase where both groups received no intervention and provides each individual's baseline level of performance. In the subsequent 6 months, called the Display phase, the feedback group had the feedback tablet display in their homes. All analyses were performed using a one-way ANOVA repeated-measures design and the Huynh-Feldt correction when data variables did not satisfy conditions of sphericity (as determined by a significant effect with Mauchly's test). The results report the significance of the interaction effect between group and phase, that is, whether the feedback group improved in performance while the control group stayed the same.

Real-time feedback improves medication-taking behaviors The introduction of the feedback display resulted in significant improvements in promptness, correctness, and time of day variance for the feedback group. Adherence increased but the change was not statistically significant. Effect sizes were calculated using the following equation for pre/post with control designs: $d=\left(\Delta \mu_{\mathrm{f}}-\Delta \mu_{\mathrm{c}}\right) / \sigma$, where

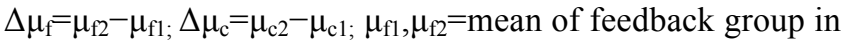
baseline and display periods respectively; $\mu_{\mathrm{c} 1}, \mu_{\mathrm{c} 2}=$ mean of control group in baseline and display periods respectively; $\sigma=$ pooled stdev at baseline [21].

\section{Adherence}

The feedback group improved in their adherence rate increasing from $94.5 \%$ in the baseline phase to $98.4 \%$ in the feedback phase (Figure 3, Left). The control group remained relatively unchanged, going from $93.7 \%$ to $92.1 \%$. The interaction between group and phase was not statistically significant $(\mathrm{F}[1,67]=2.33, \mathrm{p}=0.131)$. Participants started off highly adherent (averaging $>90 \%$ ) and with the display, reached a level that neared perfect adherence $(100 \%)$, the upper bound for a ceiling effect. In fact, all six participants with the feedback display had at least one twoweek streak of never missing a dose, something not previously achieved. The lack of statistical significance may be due to this ceiling effect. Further, Cohen's effect size value $(d=1.05)$ suggests a high practical significance.

\section{Promptness}

The promptness rate of the feedback group also significantly improved from $75.1 \%$ to $90.8 \%$ after the introduction of the feedback display, while the control group stayed relatively unchanged, going from $78.8 \%$ to $75.4 \% \quad(\mathrm{~F}[1,67]=11.40, \quad \mathrm{p}=0.0012) \quad$ (Figure 3, Center) Cohen's effect size value $(d=1.30)$ suggests a high practical significance.

\section{Correctness}

The correctness rate of the feedback group significantly increased from $94.9 \%$ to $99.2 \%$ for the feedback group after they received the feedback display while the control group remained unchanged $(95.1 \%$ to $95.0 \%)$. The interaction 

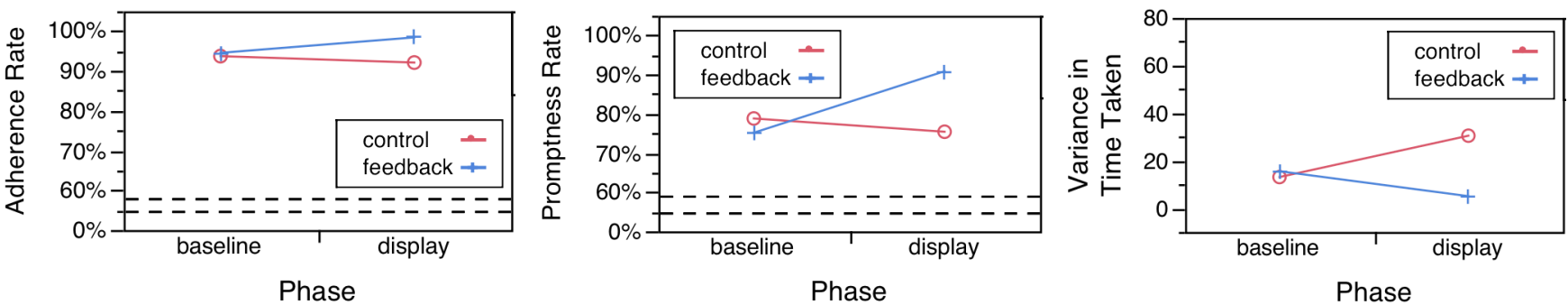

Figure 3. (Left) The feedback group improved in their adherence rate with the feedback display, but this difference was not statistically significant $(p=0.131$ ), possibly due to a ceiling effect. (Center) Promptness rates significantly increased in the feedback group while the control group did not change significantly $(p=0.0012)$. (Right) The feedback group significantly decreased in the variance in the time of day pills were taken $(\mathbf{p}<\mathbf{0 . 0 0 0 1})$ whereas the control group increased in the variance (which likely resulted from settling into the study).

effect between group and phase was statistically significant $(\mathrm{F}[1,67]=4.18, \mathrm{p}=0.0448)$. Cohen's effect size value $(d=$ 0.95 ) suggests a high practical significance.

\section{Variance in the time of day}

The variance in the time of day medications were taken (Figure 3, Right) also significantly decreased from 15.5 to 5.0 in the feedback group but increased for the control group from 13.07 to 30.47 . The interaction effect between group and phase was statistically significant $(\mathrm{F}[1,66]=17.33, \mathrm{p}<0.0001)$. Cohen's effect size value $(d=$ -2.67) suggests a high practical significance. The feedback display helped the feedback group to take their medications closer to the same time of day from one day to the next. For example, the week before he received the feedback display, participant P2 took his pills at 8:18, 9:41, 6:08, 10:11, 7:16, $11: 45$, and 8:09am. The first week with the display, P2 took his pills most consistently around $7 \mathrm{am}$, at 7:10, 6:58, 5:30, 7:30, 7:08, 7:05, and 6:55am. Interestingly, the control group increased in the variance in the time of day during this period. This can likely be attributed to participants feeling more relaxed about having their medications recorded, as they entered months 3 and 4 without feedback, and being less conscientious about taking their medications at the same time of day everyday. Thus, the feedback display seems to help maintain the level of vigilance on the consistency of medication taking rather than letting it extinguish after the initial boost from being monitored.

\section{Qualitative improvements in medication-taking behaviors}

Interviews with participants reveal that individuals used the display to remind themselves to take their medications. However, unlike reminder systems, the informational feedback from the display also enabled participants to selfregulate their medication taking behavior by 1) increasing their awareness, 2) identifying errors, and 3) confirming their memory, which helped them maintain their behavior. We describe these actions in the following paragraphs.

The process of self-regulation requires individuals to develop strategies that help overcome the obstacles to perform the target behavior. Individuals used the feedback display as a visual reminder for their medication taking. Even though participants reported that they took their medications very consistently, they all mentioned at least a few instances when they had forgotten and happened to glance at the display, which reminded them to take their medications. P01 said, "I look up and I see 'Oh! I didn't take my evening pills', so it's a nice reminder, it's not a nasty kind of thing, you know." In this case, P01 saw that the evening pill status was still gray and not yet been filled in, not some alert or visually salient change in the display. It should be noted that the feedback display did NOT show time-triggered reminders for on-time medication taking because it only updated after the individual took their medications or after the predefined late time. Even though automatically changing the status to "MISSING" after the late time can act as a trigger, the data show that participants increased both in the rate of adherence and in the rate of promptness, indicating that they mostly took their pills before the status changing to "MISSING" could act as a reminder. Nonetheless, individuals appropriated the presence of the display as a visual reminder to assist them in taking their medications.

In addition to reminding, real-time feedback also provided the individual with key information to self-regulate. One of the most common themes emerging from the interviews was about how the display provided individuals with a sense of awareness of their medication taking behaviors and habits that they had not previously realized, which is necessary for self-regulation. P01 remarked, "You know, [the display] tells me what I have done or what I haven't done. The time to take my pills would be varied. I would get them in me but the times are varied." P05 said, "It always tells you what time you get your medication then in the meanwhile sometimes it tells you when you have missed your medication. That way, it's good."

Self-regulation requires feedback about when the behavior was performed in error or inadequately to reach a sub-goal. Individuals need feedback to direct their attention to differences between their current behavior and their goal behavior. The feedback display helped individuals to identify errors in their medication-taking behavior such as opening the wrong door on the pillbox and taking another day's pills, as remarked by $\mathrm{P} 02$ who said, "[The display] lets you know if am I making mistake, like if I open another 
door one day and it came up [on the display]." The timeliness of the feedback was also important because it highlighted errors earlier than when refilling the pillbox at the end of the week. For example, P10 said, "Before, if I made a mistake and forgot it, I might not notice until the end of the week or something. [With the display] you notice it, you see it right away."

The feedback display also helped individuals to not only identify errors but also confirm correct behaviors. P02 said he made looking at the display part of his daily routine, "I find myself [checking the display] every day, to make sure I was on time or make sure I was right or make sure I did thing I was supposed to." P06 reported that it was her habit to take her pills and then look at the screen to see when it would register her pill taking, "I would take my pills and few minutes later I'm looking at [the display] to see how long it takes for the light up." Both P06 and P02 developed such strong habits to check the display to confirm their behavior that even after it was removed, they reported they still glanced over to where the display used to be.

\section{Real-time feedback improves qualitative self-efficacy}

Self-efficacy is one of the main modifiable determinants of behavior change [1]. One way to increase self-efficacy is to experience successes in performing a behavior, which can, in turn, motivate the individual to carry out the same behavior successfully again. The feedback from the tablet display can provide a signal that the medications were successfully taken promptly and correctly, which can increase self-efficacy and reinforce more consistent medication-taking behaviors.

Real-time feedback had no quantitative effect on self-efficacy Responses to each of the 13 items in the SEAMS questionnaire were assigned a value of 1 for "Not Confident", 2 for "Somewhat Confident", and 3 for "Very Confident". The values were summed up across all 13 items, for a possible total self-efficacy score of 39.

At baseline, before any intervention was introduced, participants rated themselves with very high self-efficacy, with an overall average of 33.75. This high score indicates that participants were quite confident in their abilities to overcome challenges when taking their medications.

The introduction of the feedback display did not have a significant effect on medication-taking self-efficacy. The feedback group remained relatively unchanged, going from an average self-efficacy score of 34.41 to 34.86 with the display, as did the control group, going from 33.1 to 32.1 . A repeated-measures ANOVA showed no significant interaction effect between group and phase $(\mathrm{F}[1,34]=0.406$, $\mathrm{p}=0.528)$.

These results can be interpreted in two ways: the first being that feedback had no effect on self-efficacy, the second being that our measure of self-efficacy was not sensitive enough to detect the subtle change in self-efficacy. The latter interpretation is reasonable given that our sample may have reached a ceiling effect for the SEAMS instrument, as most of them gave the highest confidence ratings to 8 (a majority) of the 13 items. A finer-grained scale may have been able to measure an effect.

Real-time feedback qualitatively improved self-efficacy To overcome the ceiling effect of the instrument, we conducted a follow-up interview and asked participants to compare their feelings of self-efficacy before they received the feedback display and their feelings with the display. For each of the 13 scenarios in the SEAMS questionnaire, we asked, "With the help of the display, do you feel more confident, less confident, or no difference about taking their medications." If the response was "more confident" or "less confident", we asked the participant to rate the degree of how much more/less confident on a scale of 1 to 5 , with 1 being "just a little" and 5 being "very much".

Of the 6 participants in the feedback group, we only could reach 5 of them for the follow-up interview because P09 was unavailable due to a family matter. None said the display made them feel less confident, only 1 participant (P01) reported that the display did not change her confidence level in any of the items, and the remaining 4 all reported that the display helped them feel more confident in their medication taking in at least one of the SEAMS scenarios. These 4 participants each reported it helped them feel more confident (average degree: 3.75 ) in taking their medication in scenario \#6 ("when no one reminds you to take your medicine"). 3 of these participants also thought the feedback display helped them feel more confident in scenario \#1 ("when you need to take several different medications per day") (average degree: 4.33) and scenario \#8 ("when your normal routine gets messed up") (average degree: 4.0).

\section{Removing Real-Time Feedback}

In this section, we investigate whether the feedback display had a lasting effect after it was removed. After 6 months with the display (the Display phase), the feedback display was removed from the homes of the feedback group. The sensor-augmented pillbox continued to track medication taking behaviors for another 2 months, which we label the Follow_up phase. We compare the medication-taking behaviors during these 2 phases. Measures of behavior were averaged for each month in these phases and a repeatedmeasures one-way ANOVA was used to find differences after the display was removed. Two control group participants were excluded from the analysis because Follow_up data was lost due a laptop disk failure (P07) and a move to another apartment (P04).

Even though real-time feedback supported a higher level of performance in the medication-taking task, the analysis revealed that the improved level of performance was not sustained after the real-time feedback was removed. Without the display, individuals significantly decreased their medication adherence (from 98.4\% to $96.2 \%$, $\mathrm{F}[1,41]=5.86, \mathrm{p}=0.0201$ ), promptness (from $90.8 \%$ to 
$83.6 \%, \mathrm{~F}[1,41]=5.8, \mathrm{p}=0.0208$ ), and correctness (from $99.2 \%$ to $97.1 \%, \mathrm{~F}[1,41]=7.08, \mathrm{p}=0.011)$. There was also a marginally significant increase in the variance of the time of day medications were taken (from 5.03 to 9.82 hours $\mathrm{F}[1,41]=3.34, \mathrm{p}=0.075)$. Post-hoc comparisons using the Tukey HSD test indicated the mean rates for adherence, promptness, and correctness were not significantly different $(p>0.05)$ from the rates in the Baseline phase before participants received the display. In other words, once the feedback display was removed, individuals dropped in performance to a level slightly (but not statistically significantly) above their baseline performance level. In the control group, none of the corresponding measures of adherence $\quad(\mathrm{F}[1,26]=1.96, \quad \mathrm{p}=0.174), \quad$ promptness $(\mathrm{F}[1,26]=4.07, \quad \mathrm{p}=0.0545), \quad$ correctness $\quad(\mathrm{F}[1,26]=0.676$, $\mathrm{p}=0.419)$, and variance $(\mathrm{F}[1,26]=0.424, \quad \mathrm{p}=0.521)$ experienced statistically significantly changes in this period, as would be expected.

\section{DISCUSSION}

Improving medication taking, like many health behaviors, is not often accomplished simply by an act of will. Adopting health behaviors requires a process that involves interplay among the individual's motivation, goals, and feedback to carry out their intentions. Feedback can play a role in helping individuals self-monitor and self-regulate.

\section{Using Real-time Feedback to Self-Regulate Behavior}

One of the research questions in this study is how feedback affects an individual's medication-taking behaviors. The results demonstrate that near real-time sensor-based feedback about medication taking behaviors presented in an ambient in-home display enabled individuals to selfmonitor their medication taking more consistently. As a result, those who received the feedback display took their prescribed doses significantly more promptly (before a user-defined "late time"), selected the right pills to take slightly more often, and took them significantly more consistently at the same time of day across multiple days. Real-time feedback allowed participants to self-regulate their medication taking by 1 ) increasing their awareness, 2) identifying errors, and 3) confirming their memory, which helped them improve their behavior.

Another important research question we addressed in this study was about what happens after individuals are cut off from feedback after using it for a relatively long period (in our case, 6 months). The results demonstrate that the gains in performance mostly disappeared after the feedback display was removed from their home. Previous studies of self-monitoring [22] have shown that the boost in performance associated with manual self-recording usually diminishes once the individual stops selfmonitoring, perhaps due to the effort and attention required for manual self-monitoring. In contrast, our approach used automated self-monitoring with sensors that require minimal effort by the user. The results show our automatic approach had a similar drop off after the feedback was removed. This indicates that the ongoing feedback itself (and not the effort of self-recording behaviors) was instrumental for boosting performance. Participants reported they had integrated the ambient feedback display into their daily routines and continued to use it for selfregulation by checking the display multiple times to see whether they took their pills earlier in the day, to identify errors, and also to confirm that their earlier medication taking had registered in the system. Without the frequent, immediate feedback from the display, individuals did not see progress on how well they were achieving the daily subgoals of taking their medications well and were not able to self-regulate their medication-taking behaviors as well.

\section{Supporting Self-Efficacy with Feedback}

Regular feedback about successes and failures in medication taking supported a qualitative increase in selfefficacy for medication taking. Even though the SEAMS questionnaire used for measuring self-efficacy was not able to detect a quantitative increase in self-efficacy due to a ceiling effect, individuals reported in interviews that the feedback display was instrumental in helping them feel more confident in taking their medications independently, when no one was available to remind them, when they had to take multiple medications in a day, and even when their daily schedule may be non-routine.

According to Social Cognitive Theory (SCT), high selfefficacy is one of the most powerful predictors of behavior change, even more powerful than demographics, personal/ environmental factors, and barriers [3]. One of the main mechanisms for building self-efficacy is successfully reaching a goal (or sub-goal) by performing the target behavior. Individuals reported that it made them feel good when they saw the status on the screen light up green, indicating that they took their medications promptly and correctly. It is also interesting to note that the display also provided negative feedback about missed or late pills. SCT predicts that self-efficacy can decrease if individuals encounter failures in performing the task. However, if individuals have the means to adjust their environment or strategies to avoid future failures, then confidence will not necessarily be eroded [1]. With our approach of providing feedback almost immediately, individuals could be made aware of a mistake, recall the circumstances from earlier in the day or yesterday that led to the mistake, and develop a strategy to avoid making the mistake again. Our study demonstrates that even individuals with high self-efficacy could (and did) improve their medication-taking behaviors even further with the addition of feedback. The results reinforce the need for behavior change interventions to support self-awareness in addition to self-efficacy. Ubiquitous sensing and feedback technologies are wellsuited to provide the data necessary for self-awareness.

\section{Design Considerations for Feedback Systems}

The results of this paper indicate that 1) feedback from a ubiquitous sensing system is useful for supporting self- 
regulation and behavior change in medication taking and 2) medication taking is an ongoing behavior that requires continued feedback beyond six months to maintain consistency in the behavior. Many other ongoing behaviors like maintaining physical activity or managing diet may also require continued feedback to sustain improvements in behaviors. Thus, it is important for designers of ubiquitous sensing systems that use feedback for behavior change, to consider how these systems can be deployed and used over the long-term to sustain behavior improvement. In evaluating dwellSense, the sensing and feedback system described in this paper, we suggest future systems to minimize the burden of sensing on the user and rely on either unobtrusive sensing or input willingly reported by the user over a long period of time. For example, the pillbox sensor used in this study was purposely designed to be used exactly as a normal pillbox, so individuals did not have to change their routines for the system to monitor their natural behaviors. An even more integrated example that similarly requires little additional demand on the user is the Proteus Digital Health Feedback System [29] that senses physiological parameters through an ingestible sensor.

We also suggest embedded feedback in a way that is easily visible and easily integrated into the individual's routines. The ambient tablet display we used in this study was always on and situated rather prominently in the user's living room where they spend most of their time. Users could not help but glance at it once in a while. As a result, glancing at it became part of their routine to support and confirm their medication taking. We speculate that if we required the user to walk up to the tablet and request a report to get feedback that they would not have viewed the feedback very often, if at all, particularly because most felt they did not need "help" in taking their medications.

The ambient (rather than highly interactive) display also affords a sense of security. Many individuals may balk at the idea of using a system to assist or persuade them in performing behaviors perceived to be simple like medication taking. However, participants in our study commonly looked at the screen just to check how they were doing and this made them feel secure, even though most did not believe they needed the feedback display to take their medications well. Technologies that monitor and provide feedback while preserving the individual's autonomy can be presented to users to support a feeling of security rather than solely as assistive technology.

Knowing when and how often to provide feedback is also an important design consideration for feedback systems. The timing of the feedback may be related to the type of behaviors the system is trying to support. Providing longerterm logs of their behaviors can provide a better view of trends in the data to indicate larger problems or good performance. However, frequent behaviors like medication taking require frequent, actionable feedback that helps users recall the reason for their mistake while it is fresh in their mind, so feedback should be provided before the next repetition of the activity (e.g., this evening's medication taking) displaces the previous instance from memory.

Finally, automated feedback systems should be considered as one tool within a larger holistic coaching strategy that addresses the many factors that lead to behavior change. Lowly activated, passive individuals may need their beliefs about medication taking first addressed through education, whereas more highly activated and motivated individuals would likely benefit more from the real-time feedback systems to adopt and maintain a desire behavior.

\section{LIMITATIONS \& FUTURE WORK}

The study examined how real-time feedback deployed into actual homes of users over a long term affected their medication-taking behaviors. One of the limitations of this study was the relatively small sample size, with a total of 12 participants, equally divided between the intervention group and control group. However, the experiment was structured with two points of comparison: an individual's own baseline (the first 2 months without intervention), and with the control group, which makes it less likely for significant effects to result from unaccounted personal factors. Similar to [23], we encountered ceiling effects due to our sample being highly adherent and reporting high efficacy at baseline. As a result, improvements in medication-taking behaviors, though often statistically significant, were somewhat small in absolute magnitude. However, effect tests indicate that the magnitudes of improvements were approximately one standard deviation, which suggests a fairly substantial increase. Self-regulation theory predicts that highlighting the discrepancy between the current and goal state can increase motivation. Feedback will show a larger discrepancy for low adherers than high adherers, which can motivate lower adherers even more, assuming they have the skills and self-efficacy to improve (via coaching, achieving success, social support, etc). In future work, we will validate this prediction without ceiling effects. Another limitation was that the control group was not a strictly no-intervention group. They received a chart showing 2 months of their medication-taking behaviors so that they could understand the type of data being collected. It is possible that seeing this chart may have reinforced their existing performance level, but it is more likely this onetime feedback had no significant lasting effect on their performance. In future work, we will investigate how less frequent reflection on longer-term data can impact selfawareness and behaviors.

\section{CONCLUSION}

Medication adherence is a self-regulatory process that requires an active process of self-monitoring and adjustments to perform medication taking consistently over time. Systems that sense and reflect back behavioral information can provide feedback for this self-regulatory process. This paper demonstrated such a system that captured medication-taking behaviors with an unobtrusive 
sensor-augmented pillbox and presented real-time feedback about the task on an in-home ambient display. This system enabled individuals to take their medications more frequently, promptly, and correctly, but these improvements diminished almost immediately without continued feedback. Feedback about when they took their medications earlier in the day enabled individuals to 1) increase their awareness, 2) identify errors sooner, and 3) confirm their memory which helped to increase their self-efficacy, sustain their performance, and preserve their autonomy without resorting to disruptive reminders. Our approach of understanding the particular self-regulatory process of the target behavior and designing a system that senses and displays timely behavioral information to close the feedback loop necessary for self-regulation, can also be applied in other behavioral domains such as improving physical activity, eating habits, and resource consumption.

\section{ACKNOWLEDGEMENTS}

This work was supported by the Robert Wood Johnson Foundation Project HealthDesign Grant RWJ67167 and National Science Foundation ERC Grant EEEC-0540865.

\section{REFERENCES}

1. Bandura, A. Self-efficacy: toward a unifying theory of behavioral change. Psychological review 84, 2 (1977), 191.

2. Bandura, A. Social cognitive theory of self-regulation. Organizational behavior and human decision processes 50 , 2 (1991), 248-287.

3. Brus, H., Van de Laar, M., Taal, E., Rasker, J., and Wiegman, O. Determinants of compliance with medication in patients with rheumatoid arthritis. Patient Education and Counseling 36, 1 (1999), 57-64.

4. Consolvo, S., Klasnja, P., McDonald, D.W., et al. Flowers or a robot army?: encouraging awareness \& activity with personal, mobile displays. In Proc. Ubicomp 2008, ACM (2008), 54-63.

5. Consolvo, S., McDonald, D.W., and Landay, J.A. Theorydriven design strategies for technologies that support behavior change in everyday life. In Proc. CHI 2009, ACM (2009), 405-414.

6. Elliott, R.A. Problems with medication use in the elderly: an Australian perspective. Journal of Pharmacy Practice and Research 36, 1 (2006), 58.

7. Erickson, T., et al. The dubuque water portal: evaluation of the uptake, use and impact of residential water consumption feedback. In Proc CHI 2012, ACM (2012), 675-684.

8. Fishkin, K.P., Wang, M., and Borriello, G. A ubiquitous system for medication monitoring. In Proc. Pervasive 2004.

9. Friedman, R.H., et al. A Telecommunications System for Monitoring and Counseling Patients With Hypertension. Am J Hypertension 9, 4 (1996), 285-292.

10. Gadkari, A.S. and McHorney, C.A. Unintentional nonadherence to chronic prescription medications. BMC Health Services Research 12, 1 (2012), 98.

11. Hayes, T.L., Cobbinah, K., Dishongh, T., et al. A study of medication-taking and unobtrusive, intelligent reminding. Telemedicine and e-Health 15, 8 (2009), 770-776.

12. Haynes, R.B., Ackloo, E., Sahota, N., McDonald, H. P., \& Yao, X. Interventions for enhancing medication adherence. Cochrane database syst Rev, 2, 2 (2008), 11.

13. Kripalani, S. Interventions to Enhance Medication Adherence in Chronic Medical Conditions. Archives of Internal Medicine 167, 6 (2007), 540.

14. Lee, M.L. and Dey, A.K. Reflecting on pills and phone use: supporting awareness of functional abilities for older adults. In Proc. CHI 2011, ACM (2011), 2095-2104.

15. Li, I. Personal informatics \& context: Using context to reveal factors that affect behavior. J. Ambient Intell. Smart Environ. 4, 1 (2012), 71-72.

16. Locke, E.A. and Latham, G.P. A theory of goal setting \& task performance. Prentice-Hall, Inc, 1990.

17. Lundell, J. et al., Continuous activity monitoring and intelligent contextual prompting to improve medication adherence. In Proc. EMBS, IEEE (2007), pp. 6286-6289.

18. Maes, S. and Karoly, P. Self-Regulation Assessment and Intervention in Physical Health and Illness: A Review. Applied Psychology 54, 2 (2005), 267-299.

19. McDonnell, P.J. and Jacobs, M.R. Hospital admissions resulting from preventable adverse drug reactions. The Annals of pharmacotherapy 36, 9 (2002), 1331-1336.

20. McGee-Lennon, M.R., Wolters, M.K., and Brewster, S. User-centred multimodal reminders for assistive living. In Proc. CHI 2011, ACM (2011), 2105-2114.

21. Morris, S.B. Estimating effect sizes from pretest-posttestcontrol group designs. Organizational Research Methods, 11, 2, (2008), 364-386.

22. Nelson, R.O. and Hayes, S.C. Theoretical Explanations for Reactivity in Self-Monitoring. Behavior Modification 5, 1 (1981), 3-14.

23. De Oliveira, R., Cherubini, M., and Oliver, N. MoviPill: improving medication compliance for elders using a mobile persuasive social game. In Proc. Ubicomp 2010. ACM (2010), 251-260.

24. Risser, J., Jacobson, T.A., and Kripalani, S. Development and psychometric evaluation of the Self-efficacy for Appropriate Medication Use Scale (SEAMS) in lowliteracy patients with chronic disease. Journal of nursing measurement 15, 3 (2007), 203-219.

25. Rogers, Y., Hazlewood, W.R., Marshall, P., Dalton, N., and Hertrich, S. Ambient influence: can twinkly lights lure and abstract representations trigger behavioral change? In Proc. Ubicomp 2010, ACM (2010), 261-270.

26. Williams, G., Rodin, G., Ryan, R., Grolnick, W. and Deci, E. Autonomous regulation and long-term medication adherence in adult outpatients. Health Psychology, 17, 3 (1998), 269.

27. Zhu, L.L., Zhou, Q., Yan, X.F., Zeng, S. Optimal time to take once-daily oral medications in clinical practice. International Journal of Clinical Practice, 62 (2008), 1560.

28. http://www.vitality.net/glowcaps.html.

29. http://proteusdigitalhealth.com/. 